\title{
Burning Rate of Liquid Supplied Through a Wick
}

\author{
Y.-X. TAO* and M. KAVIANY \\ Department of Mechanical Engineering and Applied Mechanics, University of Michigan, \\ Ann Arbor, MI 48109
}

\begin{abstract}
Liquid fuel supplied through ceramic or metallic wicks is commonly used as a simulant for burning of planar liquid or solid surfaces. When the liquid flow through the wick is at least partly controlled by capillarity, the partially exposed wick matrix at the burning surface changes the concentration distribution adjacent to this surface. Here we show that when the characteristic length of the exposed matrix is not significantly smaller than the convection boundary-layer thickness, the burning rate from the wick deviates (being smaller for low thermal conductivity wicks) from that for planar liquid surfaces. The study is guided by experiments using a ceramic wick, by a one-dimensional gas-side diffusion analysis, and by a wick-side two-phase flow and heat transfer analysis.
\end{abstract}

\section{NOMENCLATURE}

$b \quad$ dependent variable in Eq. 2

$B$ mass transfer number

Bo Bond number, $\left(\rho_{l}-\rho_{g}\right) g \mathrm{~d}^{2} / \sigma$

$d$ particle diameter $(\mathrm{m})$

$D_{n v} \quad$ binary mass diffusivity $\left(\mathrm{m}^{2} / \mathrm{s}\right)$

$f$ saturation function in the capillary pressure equation

$h \quad$ height of the liquid column (m)

$i \quad$ enthalpy $(\mathrm{J} / \mathrm{kg})$

$i_{f g} \quad$ enthalpy of evaporation $(\mathrm{J} / \mathrm{kg})$

$g$ gravitational constant $\left(\mathrm{m} / \mathrm{s}^{2}\right)$

$k$ thermal conductivity $(\mathrm{W} / \mathrm{m}-\mathrm{K})$

$K$ permeability $\left(\mathrm{m}^{2}\right)$

$K_{r l} \quad$ liquid relative permeability

$K_{r g} \quad$ gas relative permeability

$L$ block height (m)

$l \quad$ block length $(\mathrm{m})$

$\dot{m}^{\prime \prime} \quad$ mass flux $\left(\mathrm{kg} / \mathrm{m}^{2}-\mathrm{s}\right)$

$\dot{m}$ mass flow rate $\left(\mathrm{kg} / \mathrm{m}^{2}\right)$

$n$ normal direction

$p \quad$ pressure $\left(\mathrm{N} / \mathrm{m}^{2}\right)$

$\dot{q}^{\prime \prime} \quad$ heat flux $\left(\mathrm{W} / \mathrm{m}^{2}\right)$

$Q$ heat of reaction $(\mathrm{J} / \mathrm{kg})$

$R$ gas constant for gas mixture $(\mathrm{J} / \mathrm{kg}-\mathrm{K})$

$\operatorname{Re}_{1} \quad$ Reynolds number, $u_{\infty} l / \nu$

$s$ saturation, $\epsilon_{l} / \epsilon$

$S \quad\left(s-s_{\text {im }} /\left(1-s_{\text {im }}\right)\right.$
$T \quad$ temperature (K)

$u$ velocity $(\mathrm{m} / \mathrm{s})$

$u_{\infty}$ ambient, free-stream velocity $(\mathrm{m} / \mathrm{s})$

$x$ horizontal coordinate axis (m)

$y$ mass fraction of the vapor

$y_{n} \quad$ mass fraction of the noncondensible gas

$z, Z$ coordinate axis opposite to gravity (m)

\section{Greek Symbols}

$\alpha \quad$ thermal diffusivity, $k_{s l} /(\rho c)_{l}\left(\mathrm{~m}^{2} / \mathrm{s}\right)$

$\beta \quad$ stoichiometric fuel-air ratio

$\delta \quad$ boundary layer thickness (m)

$\Delta \quad$ surface roughness (m)

$\epsilon$ porosity

$\Phi \quad$ defined in Eq. 12

$\lambda \quad$ mean free path length of gaseous molecules (m)

$\mu \quad$ viscosity $(\mathrm{Pa}-\mathrm{s})$

$\xi \quad$ length scale variable $(\mathrm{m})$

$\rho$ density $\left(\mathrm{kg} / \mathrm{m}^{3}\right)$

$\sigma \quad$ surface tension $(\mathrm{N} / \mathrm{m})$

$\tau$ tortuosity

\section{Superscripts}

\section{- $\quad$ average}

* evaluated at the liquid surface

, evaluated at the bottom of the boundary layer

\section{Subscripts}

*Present address: Department of Mechanical Engineering, University of Saskatchewan, Saskatoon, Saskatchewan, Canada S7N 0W0. 
1 evaluated at the surface with flame

$\infty \quad$ ambient

app apparent

$b \quad$ burning

$c$ capillary

eff effective

$f \quad$ liquid film

$g$ gaseous phase

im immobile or irreducible

$l$ liquid phase

$n$ noncondensible

$r$ relative

ref reference

$s \quad$ solid phase

sat saturation

$v \quad$ vapor

\section{INTRODUCTION}

In addition to their applications in heaters, wicks are often used in experiments that simulate the burning of liquid and solid surfaces [1-3]. In these applications and under steady-state conditions, the liquid is supplied to the burning surface. It is generally assumed that a thin liquid film is present on this surface. If capillarity is used for the flow of the liquid, then a gradient of the local liquid saturation exists. Local liquid saturation is defined as the fraction of the local void volume in the wick that is occupied by the liquid. Gravity can assist or oppose the flow depending on the surface orientation. The presence of this liquid saturation gradient requires that at the burning surface the wick is not completely covered by a continuous planar layer of liquid. This makes for a nonsmooth surface where the wick matrix is exposed at various locations. Generally, these surface roughnesses do not substantially influence the fluid dynamics near the surface, especially in the burning of liquids, whereas in other diffusion flames, mass diffusion is the dominant mechanism adjacent to the burning surface. It is expected that this diffusion at and adjacent to the burning surface can be noticeably influenced by the surface roughness resulting from partial surface liquid saturation. Of course, the influence is noticeable only when the length scale associated with the exposed dry patches is much larger than the mean free path of the evaporated fuel molecules [4-6].

In a transient experiment [7], a cubic, metallic wick was initially fully saturated with a liquid fuel, and then one vertical side was exposed to otherwise stagnant air and ignited. As the fuel was consumed on this surface, the liquid saturation distribution changed in the wick and gradually the surface saturation was lowered to a threshold value beyond which the flame quenched. Although the time-averaged burning rate was nearly that predicted by [8] for a fully liquid covered planar surface, the burning rate decreased monotonically (except for a short period when the wick was warming up and during which the burning rate increased with time). Whether this monotonic decrease of burning rate was associated with a decrease in the surface liquid saturation was not substantiated, however, because of the limited accuracy of the experiment. It should also be mentioned that throughout the experiment, the surface temperature was the saturation temperature of the liquid fuel and that the flame was stable up to shortly before quenching. This indicates that as the surface liquid saturation decreases, the liquid distribution over the surface (which is partially covered with liquid) changes, leading to a decrease in the burning rate. Inversely stated, burning is stable even if the liquid flow rate is below that corresponding to a flooded (thin liquid film) surface.

In the following, we examine burning of the fuel supplied through a wick at flow rates from flooding to lower but stable rates where the surface will be partially saturated. We report the results of experiments performed where the fuel supply through the wick is controlled. Then, we analyze the mass diffusion adjacent to this surface using a simple model to show that the surface liquid saturation, i.e., the distribution of the liquid in the surface pores, develops in a manner which leads to smaller burning rates as the surface saturation decreases. To do this, an estimate of the saturation distribution in the wick, including the surface saturation, is needed. This is provided by analysis of two-phase flow in the wick. We note that the study does not aim to predict the burning rate of liquid supplied through wicks, but uses experimentally measured burning rates to point out that stable burning can result, although at lower rates, when the surface is not flooded. This is especially important in the design of experiments using liquid supplied through wicks as a simulant for liquid or solid surfaces. 
Secondly, we show that the extra impedance that the vapor encounters when leaving the liquid surface explains the decrease in the burning rate when the surface saturation decreases (impedance is caused by the passages created by partial exposure of the wick surface).

The experiment uses a ceramic wick placed flush to the bottom of a wind tunnel and connected from below to a liquid ethanol reservoir. The reservoir is cooled and is under controllable hydrostatic pressure. The experimental results are in the form of mass flow rate as a function of the hydrostatic pressure, i.e., $\dot{m}(h)$. This includes the case of surface flooding for which a prediction exists [9]. The air velocity in the tunnel is varied. Regimes where buoyancy effects are significant (low velocities) or where the flame is nearly blown off (high velocities) are avoided. This results in a small range of velocity between these regimes. A single velocity is chosen in this stable and inertia-dominated range for study of the surface saturation effect. The temperature in the wick is also measured at various locations. This temperature distribution is used as an aid to determine the distribution of noncondensible gas (nitrogen) that exists in the wick along with the fuel vapor, since a fraction of the pore volume is not occupied by liquid.

Finally, we emphasize that the treatment of vapor diffusion near the gas side of the surface and the treatment of heat and two-phase flow in the wick are simplified, one-dimensional treatments. Therefore, the results only are expected to be qualitative and the trends suggestive. Also, our gas-side analysis considers vapor diffusion only and does not address heat transfer from the gas to a partially liquid covered surface. Since this heat is transferred directly to the liquid and also indirectly via the exposed solid, there is expected to be a difference in the burning rate of metallic and ceramic wicks once the wick is exposed (i.e., when the surface liquid saturation is less than unity).

\section{EXPERIMENT}

The experimental setup is shown in Fig. 1. The wick is a porous ceramic $(60 \%$ silica and $40 \%$ alumina, made by Hamilton Porcelain in Brantford, ONT) block of $4.3 \times 4.3 \times 3.0 \mathrm{~cm}$. The $3.0-\mathrm{cm}$ side is vertical. Liquid ethanol flows through the wick from the reservoir placed underneath the wick, by capillary and hydrostatic pressures. The absolute permeability $K\left(\mathrm{~m}^{2}\right)$ of the wick was measured using single-phase flows of liquid ethanol and air. The procedure involves standard flow and pressure measurement [10]. Because the air viscosity is small, the Darcy regime was not realized, and the permeability was determined using the Ergun extension of the Darcy law [10]. The results for $K$ are shown in Figs. $2 a$ and $2 b$. Based on these results, the absolute permeability was taken to be $8.5 \times$ $10^{-12} \mathrm{~m}^{2}$. The porosity of the block was determined by comparing the measured mass of the dried wick with the measured mass of the wick soaked in a beaker containing ethanol. The measurement was repeated for several temperatures. Figure 2c shows the measured apparent porosity $\epsilon_{\text {app }}$. Its asymptotic value $\epsilon$ as $T_{\text {sat }}(351.5 \mathrm{~K})$ is used in the analysis. The air velocity in the tunnel was measured with a hot wire anemometer: little disturbance was found for velocities less than 1.8 $\mathrm{m} / \mathrm{s}$. Figure $2 \mathrm{~d}$ shows typical velocity histories measured a few centimeters above the wick. A liquid-level-controller (type A-2297, Oil-Rite., Manitowac, WI) was used to maintain the hydrostatic height $h$ (shown in Fig. 1). The flow rate was measured from the volume change in a set of graduated tubes. The liquid ethanol in the reser-

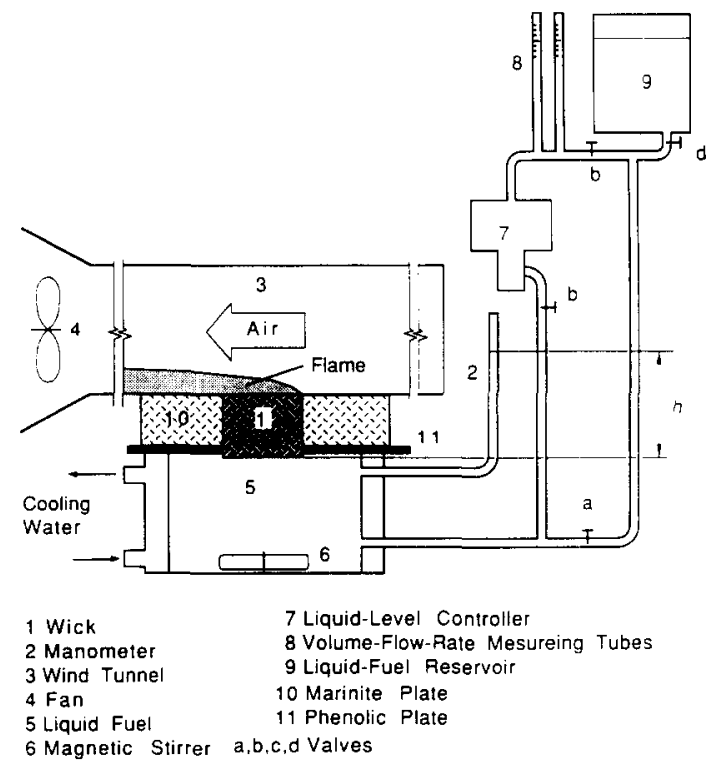

Fig. 1. A schematic of the apparatus. 


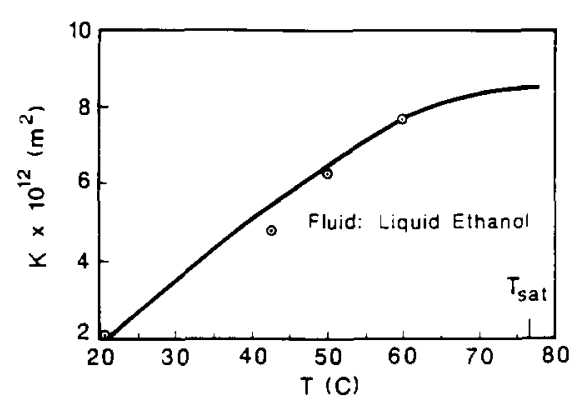

(a)

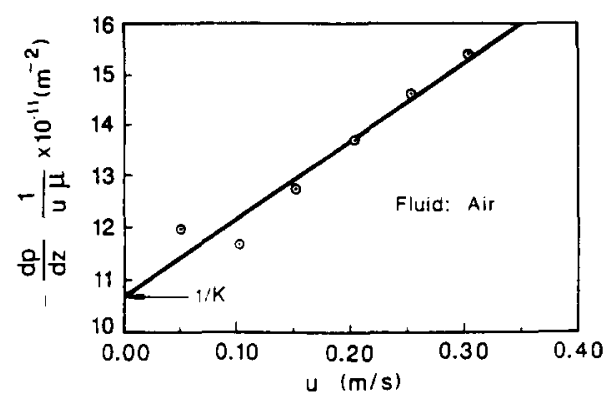

(b)

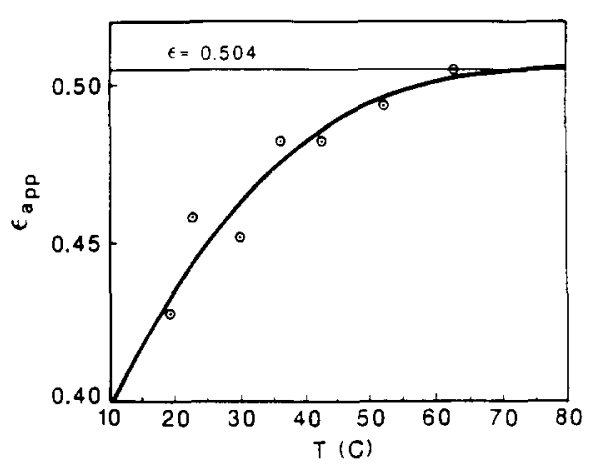

(c)

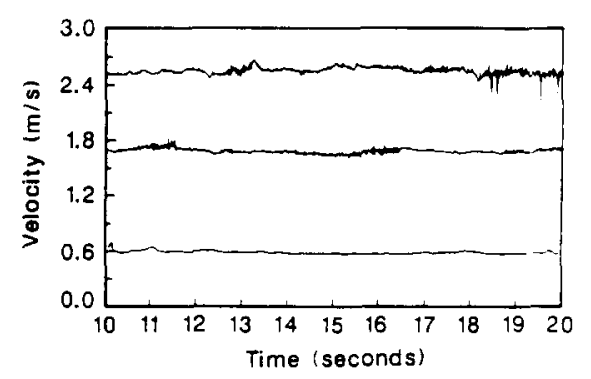

(d)

Fig. 2. (a) The measured absolute permeability using liquid ethanol, (b) using air, (c) the measured porosity, and (d) the measured ambient air velocity. voir was cooled and kept at the room temperature $T_{0}$. This is necessary in order to avoid evaporation inside the reservoir.

\section{MASS DIFFUSION ADJACENT TO PARTIALLY LIQUID SATURATED SURFACES}

Before considering the burning of liquid fuel on partially covered wick surfaces, let us consider a similar process as it occurs in the well-studied convective drying of porous media. It has been established that when the characteristic length of the liquid discontinuity on the surfaces is not very much larger than the mean free path of the gaseous molecules, $\lambda$, then the dry patches are practically covered by the molecules drifting from the wet patches. This characteristic length is the size of the dry or wet patches or the depth of indentations (valleys) $\Delta$ measured from the top of the solid to the liquid level. There is an additional requirement that is generally satisfied in drying processes. This requirement is that the concentration boundary-layer thickness, $\delta$, must be much larger than $\Delta$, i.e., $\Delta / \delta \rightarrow 0$. This results in no change in the evaporation rate (or drying rate) as the surface saturation decreases. These trends continue until the surface becomes completely dry. Then, drying proceeds as an interface separating the dry and wet regions moving into the porous media. Therefore, when the surface liquid saturation $s_{1}$ is not zero, $\Delta / \lambda$ is not very large and $\Delta / \delta \rightarrow 0$, then the evaporation rate is a constant called the constant drying rate.

The bounds for maximum values of $\Delta / \lambda$ and $\Delta / \delta$ for which the evaporation rate is nearly constant have been studied in Ref. 6. The model in Ref. 6 is based on liquid patches (having a hemispherical shape) placed on a planar surface. However, the line of reasoning used below is independent of which phase (liquid or solid) protrudes. The approach in Ref. 6 uses the addition of the Knudsen (molecular) diffusion and the Fickian diffusion (in series) over $\Delta$ and the Fickian diffusion over $\delta-\Delta$. The mean free path [11] is

$$
\lambda=\frac{1.051 k_{B} T}{2^{1 / 2} \pi d_{m}^{2} p},
$$

where the molecule diameter $d_{m}$ is about $4 \times$ 
$10^{-10} \mathrm{~m}, T$ and $p$ are saturation temperature and pressure, and $k_{B}$ is the Boltzmann constant. While for low temperature (small vapor pressure drying), $\lambda$ is relatively large, for burning where the surface is at the saturation temperature and pressure, $\lambda$ is relatively small $\left(\lambda=7 \times 10^{-8} \mathrm{~m}\right.$ for ethanol at saturation conditions). The model used in Ref. 6 shows that for small $\Delta / \delta(\Delta / \delta<$ 100 ), the effect of surface liquid saturation $s_{1}$ on the evaporation rate is not significant until $s_{1}<$ 0.3 . For $\Delta / \delta=100$, a $\Delta$ of $7 \mu \mathrm{m}$ is needed for ethanol combustion. This shows that if the wick is chosen with an average pore or particle diameter of less than $7 \mu \mathrm{m}$ with ethanol as the fuel, the surface does not need to be flooded. In practice, consolidated porous media (e.g., sintered powders) made of particles of this size, offer a very large resistance to liquid flow, and capillary-hydrostatic feeding becomes almost impossible. In our experiments, for a reasonable hydrostatic height, we had to settle for $K=8.5 \times 10^{-12} \mathrm{~m}^{2}$ which, when used along the Carman-Kozeny relation assuming particles of diameter $d$, i.e.,

$$
K=\frac{\epsilon^{3}}{180(1-\epsilon)^{2}} d^{2},
$$

gives $d=55 \mu \mathrm{m}$. Therefore, we expect the surface saturation to be important. We should consider the prediction of Ref. 6 as an estimate since no rigorous analysis of Knudsen-Fickian diffusion at the surface is available. However, this prediction suggests that the evaporation rate can noticeably decrease when $s_{1}$ is small. In order to examine and estimate this reduction in the evaporation rate, a simple, one-dimensional model (perpendicular to the air flow) is devised that allows for the resistance offered to the diffusion of the vapor through the passages created by the exposure of the wick. The model is based on the available, boundary-layer treatment of combustion [12].

The model needs, as a parameter, the location of the liquid surface in each passage. This meniscus is assumed to be planar for simplicity (the actual meniscus is capillary-controlled and is curved). The location of the liquid surface in each passage is determined from the surface liquid saturation obtained through the analysis outlined in section on surface liquid saturation.
In addition to the assumptions given in [12], the surface model is based on the following assumptions:

a. The porous surface is approximated by a layer of packed spheres with the simple cubic arrangement.

b. The effect of capillarity on the meniscus contour is neglected. The partial saturation of the surface is represented by a receding of flat liquid surface.

c. Local thermal equilibrium exists between the liquid and solid phases in the unit cell.

d. The flame is stable, i.e., the flame location is independent of time.

The surface configuration based on assumptions (a) and (b) is used to simulate the wick. Assumption (b) is not fully justifiable because the Bond number, $B o=\left(\rho_{l}-\rho_{g}\right) g \mathrm{~d}^{2} / \sigma$, is not large enough. On the other hand, computation of the three-dimensional meniscus contour is rather computationally intensive. For these computations to be of reasonable accuracy the dynamics of the contact angle and the liquid motion within the pore must be included in the analysis. Twodimensional, static menisci have been computed in $[4,5]$ using the Young-Laplace formulation. Comparison with experimental results [4] shows that due to uncertainty in the specification of the contact angle (depending on the procedure used in the initial filling of the ceramic with the liquid, the advancing or receding contact angle must be used and these are substantially different), and also due to the evaporation and some liquid motions, the Young-Laplace static formulation is not valid. The experimental results [4] show that the measured capillary pressure is smaller than the static prediction. This supports the assumption of planar menisci made in our analysis. Assumption (c) neglects the difference in the thermal conductivity between the solid and liquid and is justified for nonmetallic wicks. Also, the experiment shows that the wick surface temperature is very close to the saturation temperature at the atmospheric pressure [7, 18]. It is, therefore, reasonable to assume that the temperature gradient in a surface pore (modeled by a unit cell here) is negligible, which leads to the decoupling of heat and mass transfer. The last assumption excludes the discussion of flames near extinction condi- 


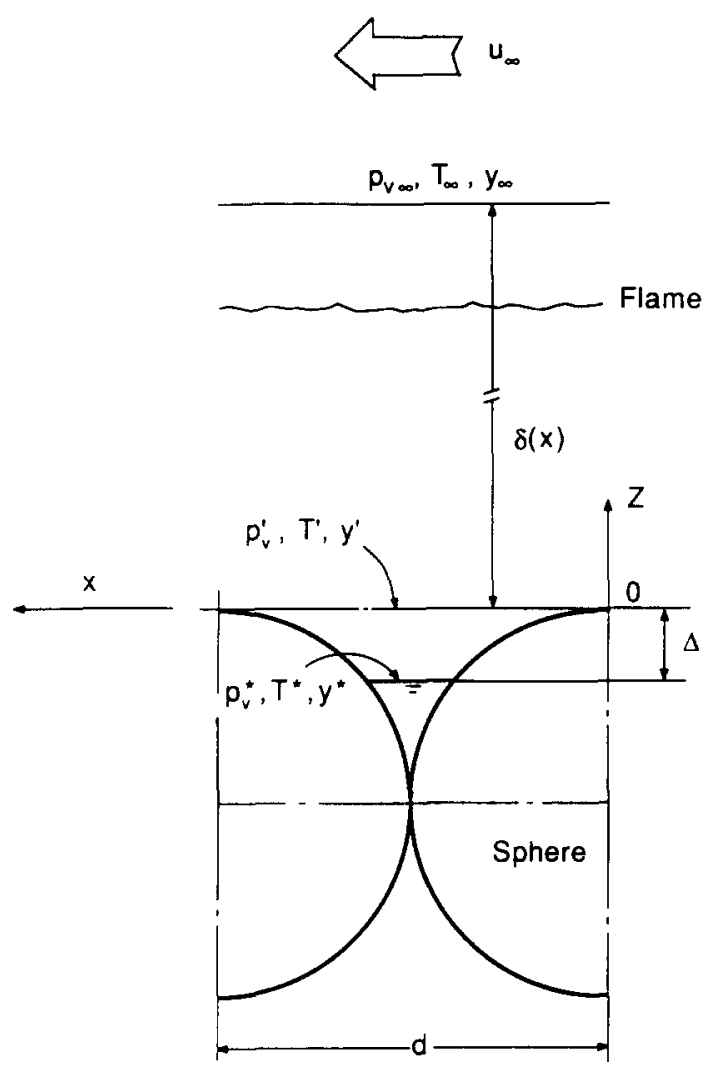

Fig. 3. The schematic of the problem of diffusion and convection from a partially wetted surface. The unit cell with the spherical particle are also shown.

tions. Figure 3 is a schematic of the problem: a unit cell of the surface layer and the edge of viscous boundary layer located above. The thickness of the boundary layer is $\delta$. A Schmidt number of unity is assumed, giving equal momentum and concentration boundary-layer thicknesses. We also assume a Prandtl number of unity. The vapor evaporates from the liquid surface at its partial pressure $p_{v}^{*}$, saturation temperature $T^{*}$, and mass fraction $y^{*}$ and diffuses through the distance $\Delta$ to a hypothetical surface where $Z=0$ and the vapor partial pressure, temperature, and mass fraction are denoted by $p_{v}^{\prime}, T^{\prime}, y^{\prime}$, respectively. Then, the vapor diffuses and convects through the boundary layer into the ambient $\left(p_{\infty}, T_{\infty}, y_{\infty}\right)$. Since we expect $\delta / \Delta \gg 1$, the boundary-layer thickness is measured from $Z=0$ (this requires that we confine the effect of the surface roughness to the thermal and concentration boundary layers). The surface saturation of the liquid is defined as

$$
s=\frac{\text { volume of liquid in the void }}{\text { total volume of the void }} .
$$

From the geometry in Fig. 3, we have the following

$$
s_{1}=1-\frac{\Delta / d}{1-\pi / 6}\left[\frac{\pi}{3}\left(\frac{\Delta}{d}\right)^{2}-\frac{\pi}{2}\left(\frac{\Delta}{d}\right)+1\right] .
$$

The problem can be divided into two parts: (a) convection and diffusion through the boundary layer, and (b) diffusion only in the layer made of the exposed particles (here called the $\Delta$ domain). Since $\delta / \Delta \gg 1$, convection within the $\Delta$-domain is negligible. As $\Delta \rightarrow 0$ (i.e., $s_{1}=1$ ), the problem reduces to (a) only.

\section{Stagnant Film Problem}

The one-dimensional transport equation for a stagnant film has the form [12]

$\rho u \frac{d b}{d Z}=\rho D \frac{d^{2} b}{d Z^{2}} \quad$ for $0 \leq Z \leq \delta$,

where $b$ is defined in Ref. 12. The boundary conditions are

$\rho u\left(\frac{d b}{d Z}\right)=\rho_{\text {sat }} u_{\text {sat }}=\rho u$,

$b(Z=0)=b_{o}$,

$b(Z=\delta)=b_{\delta}$.

The mass transfer flux can be obtained from the solution of the above equation and is

$\dot{m}^{\prime \prime}=\rho_{\text {sat }} u_{\text {sat }}=\frac{\rho D}{\delta} \ln (1+B)$,

where $B$ is the Mass Transfer Number. For complete combustion,

$B=\frac{\beta y_{O, \infty}+y_{\mathrm{sat}}}{1-y_{\mathrm{sat}}}=\frac{c_{p}\left(T_{\infty}-T_{\mathrm{sat}}\right)+\beta y_{O, \infty} Q}{i_{f g}}$,

where $\beta$ is the stoichiometric fuel-air ratio and $y_{O, \infty}$ is the mass fraction of oxygen in the air 
stream. It should be mentioned that $B$ can be modified to include the heat transferred through the liquid and solid by simply adding an additional term $Q_{c}$ to $i_{f g}$ [9]. Our calculation, based on the experimental data, indicates that adding this term has a negligible influence on the predicted mass transfer rate, as is discussed later.

The total mass transfer rate along the wick length $l$ is

$\dot{m}=N d l \frac{\rho D}{\bar{\delta}} \ln (1+B)$,

where $N$ is the number of cells along the third dimension perpendicular to the $x$ axis and

$\frac{1}{\bar{\delta}}=\frac{1}{l} \int_{0}^{l} \frac{d x}{\delta(x)}$.

For $\Delta=0\left(s_{1}=1\right.$, i.e., fully liquid-covered surface), we have

$\dot{m}_{f}=N d l \frac{\rho D}{\bar{\delta}} \ln \left(1+B^{*}\right)$.

If we use the same approximation used in Ref. 12 to fit the Emmons prediction [9], i.e., we divide the left side of the above equation by $B^{0.15} / 2$, we have

$\dot{m}_{f}=N d l \frac{\rho D}{\bar{\delta}} \frac{\ln \left(1+B^{*}\right)}{B^{* 0.15} / 2}$.

Furthermore, by extending this treatment to cases where the surface saturation is less than unity, the normalized burning rate for any surface roughness $(0<\Delta<d)$ can be expressed as

$$
\frac{\dot{m}}{\dot{m}_{f}}=\frac{\ln \left(1+B^{\prime}\right)}{\ln \left(1+B^{*}\right)}\left(\frac{B^{*}}{B^{\prime}}\right)^{0.15}
$$

where $B^{\prime}$ and $B^{*}$ are defined in the same way as by Eq. 5 with $T_{\text {sat }}$ and $y_{\text {sat }}$ being replaced by $T^{\prime}$ (or $T^{*}$ ) and $y^{\prime}\left(\right.$ or $y^{*}$ ) respectively, and $\dot{m}_{f}$ is the Emmons prediction for thin liquid films.

\section{Diffusion in a Variable-Area Passage ( $\Delta$ Domain)}

When the surface liquid saturation is less than unity, the solid matrix becomes exposed and the evaporated vapor has to diffuse from the liquid surface to the boundary layer. Then, the state of the gas mixture at location $Z=0$ (represented by the vapor properties, $p_{v}^{\prime}, T^{\prime}$, and $\left.y^{\prime}\right)$ is different than at the liquid surface $\left(p_{v}^{*}, T^{*}, y^{*}\right.$, etc. $)$ due to the additional diffusion length $\Delta$. For diffusion only, the mass transfer rate per unit cell (see Fig. 3) can be expressed as

$$
\begin{aligned}
& \dot{m}^{\prime}=-A_{l} \rho D \frac{d y}{d \xi}=\text { constant } \\
& \qquad \text { for } 0 \leq \xi \leq \Delta,
\end{aligned}
$$

where $\xi=Z+\Delta$ ( $\xi$ varies from 0 to $\Delta$ when $Z$ varies from $-\Delta$ to 0 ), and $A_{l}$ is the liquid surface area and is a function of $\Delta$ (or $s_{1}$ in Eq. 1) and is given by

$$
\begin{aligned}
& A_{l}=\pi d^{2}\left[\left(\frac{\xi}{d}\right)^{2}+c_{1}\left(\frac{\xi}{d}\right)+c_{2}\right], \\
& c_{1}=1-2 \frac{\Delta}{d} ; \quad c_{2}=\frac{1}{\pi}-\frac{\Delta}{d}+\left(\frac{\Delta}{d}\right)^{2} .
\end{aligned}
$$

The boundary conditions are

$y(\xi=0)=y^{*}$,
$y(\xi=\Delta)=y^{\prime}$

$y(\xi=\Delta)=y^{\prime}$.

Integration of Eq. 10 yields

$\dot{m}^{\prime}=\rho D d \frac{y^{*}-y^{\prime}}{\Phi}$,

where

$$
\begin{gathered}
\Phi=\frac{2 / \pi}{\sqrt{\frac{4}{\pi}-1}}\left[\tan ^{-1}\left(\frac{1}{\sqrt{\frac{4}{\pi}-1}}\right)\right. \\
\left.-\tan ^{-1}\left(\frac{1-2 \Delta / d}{\sqrt{\frac{4}{\pi}-1}}\right)\right] .
\end{gathered}
$$

The total mass transfer rate along the surface of length $l$ (in the flow direction) is

$$
\dot{m}=N \frac{l}{d} \rho D d \frac{y^{*}-y^{\prime}}{\Phi} \text {. }
$$

When divided by Eq. 8 , the above equation becomes

$$
\frac{\dot{m}}{\dot{m}_{f}}=\frac{y^{*}-y^{\prime}}{\Phi d / \bar{\delta}} \frac{B^{*^{0.15}} / 2}{\ln \left(1+B^{*}\right)} \text {. }
$$


Equation 14 gives a relation between the normalized mass transfer rate and the surface saturation ( $\Phi$ is a function of $s_{1}$ through Eqs. 1 and 12) for the given thin-liquid-film properties $B^{*}$ and $y^{*}$, and $\bar{\delta} / d$. However, we still have an unknown, the mass fraction of the vapor at $Z=$ $0, y^{\prime}$. We can use the solution obtained from the stagnant-film analysis (Eq. 9) to obtain $y^{\prime}$, since Eq. 9 contains an unknown $B^{\prime}$ which is also a function of $y^{\prime}$ (Eq. 5). This is done by using a matching condition based on mass conservation.

\section{Solution for $\dot{m} / \dot{m}_{f}$ as a Function of $s_{1}$}

The matching condition for the solutions from the two domains is

$\dot{m}_{\Delta \text { domain }}=\dot{m}_{\mathrm{b}, 1}$.

From Eqs. 9, 14, and 15, we have

$$
\begin{aligned}
\frac{\dot{m}}{\dot{m}_{f}} & =\frac{\ln \left(1+B^{\prime}\right)}{\ln \left(1+B^{*}\right)}\left(\frac{B^{*}}{B^{\prime}}\right)^{0.15} \\
& =\frac{y^{*}-y^{\prime}}{\Phi d / \bar{\delta}} \frac{B^{* 0.15} / 2}{\ln \left(1+B^{*}\right)} .
\end{aligned}
$$

This gives the relation between the evaporation rate and the saturation with the ratio $\bar{\delta} / d$ as a parameter, i.e.,

$\dot{m} / \dot{m}_{f}=f\left(s_{1}, \bar{\delta} / d\right)$.

In order to obtain the above relation, we rewrite Eq. 16 as

$\ln \left(1+B^{\prime}\right)=\frac{y^{*}(1-\eta)}{\Phi d / \bar{\delta}} \frac{B^{\prime 0.15}}{2}$,

where $\eta=y^{\prime} / y^{*}$. Inserting Eq. 5 into the above equation yields

$$
\begin{aligned}
\eta=1 & -2 \frac{d}{\bar{\delta}} \frac{\Phi}{y^{*}}\left(\frac{1-y^{*} \eta}{\beta y_{O, \infty}+y^{\prime} \eta}\right)^{0.15} \\
& \times \ln \left(\frac{1+\beta y_{O, \infty}}{1-y^{*} \eta}\right) .
\end{aligned}
$$

At a given $\bar{\delta} / d$, for an independent variable $s_{1}$, $\Delta / d$ can be found from Eq. 1 . Noting that

$$
y^{*}=\frac{\rho_{v}^{*}}{\rho} \simeq \frac{p_{v}^{*}}{p},
$$

where $p_{v}^{*}$ is the vapor pressure and obeys Clapeyron equation, $T^{*}$ and $y^{*}$ can then be solved for by iteration on Eqs. 18 and 5, and $\eta$ can be obtained by iterating on Eq. 17. Therefore, a dependent variable $\dot{m} / \dot{m}_{f}$ is determined from Eq. 16 using $\eta$ and $y^{*}$.

In the above, we presented an analysis of convection and mass diffusion in the stagnant film (considering the average boundary-layer thickness) in series with mass-diffusion in a surface indentation ( $\Delta$ domain). Mass conservation is applied to the two solutions, which yields a general relation between the normalized burning rate and the surface saturation in an implicit form, Eq. 16. This equation can be used to qualitatively predict the effect of the surface saturation of the liquid on the burning rate for wicks with low thermal conductivities.

\section{SURFACE LIQUID SATURATION}

In order to determine the surface liquid saturation required for the analysis of the mass diffusion adjacent to the surface, the flow of liquid ethanol in the wick caused by capillary and hydrostatic forces is analyzed. The momentum equations (for the liquid and vapor phases) are coupled with the energy equation because the vapor pressure distribution depends on the temperature distribution (through Clapeyron-Kelvin equation for the thermodynamic equilibrium states). Figure 4 gives the boundary conditions used. The liquid saturation at the lower surface of the wick $(s(z=0)=$ $s_{o}$ ) is not known. This follows, unless relatively high flow rates or high temperatures (but less than $T_{\text {sat }}$ ) are used, because the nonwetting phase entrapped in the pores adjacent to this lower surface cannot be removed. The transition from a plain medium (the reservoir) to a porous medium, and this discontinuity in the saturation, have been addressed by Leverett [13]. Although we did not make local liquid saturation measurements, our analysis of flow through the wick also suggests that $0.8<s_{o}<1$, i.e., a jump in the saturation occurs in transition from the reservoir to the wick.

The volume-averaged conservation equations for thermal energy, linear momentum, and mass for one-dimensional, steady-state, two-phase flow in porous media are found by the proper reductions of the equations given in [14]. The energy 

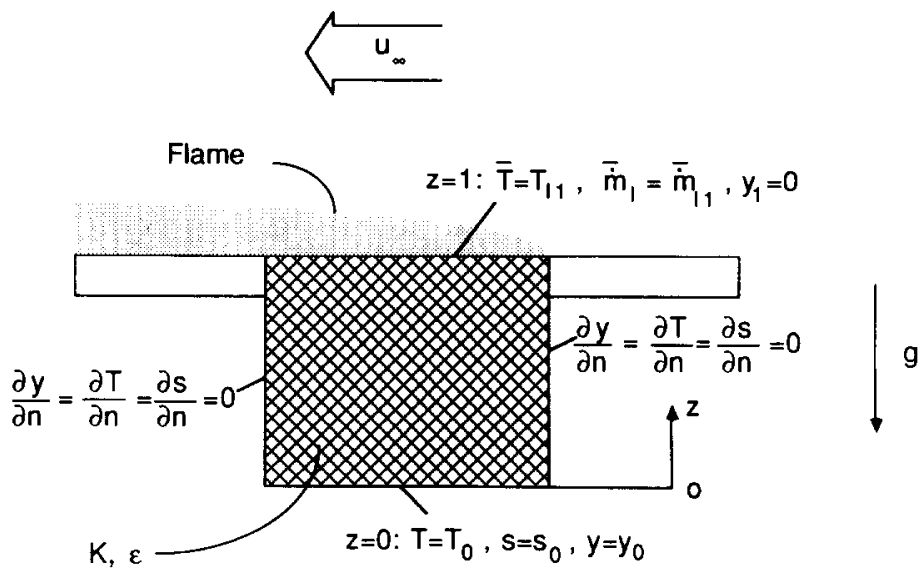

Fig. 4. The boundary conditions used in solving for the surface liquid saturation.

and continuity equations are

$$
\begin{aligned}
& \frac{d}{d z}\left[(\rho u i)_{l}+(\rho u i)_{v}\right]=\frac{d}{d z}\left(k_{\text {eff }} \frac{d T}{d z}\right), \\
& \frac{d}{d z}\left(\rho_{l} u_{l}+\rho_{v} u_{v}\right)=0 .
\end{aligned}
$$

Now letting

$\dot{m}^{\prime \prime}=\rho u$,

and integrating Eqs. 19 and 20 once, the following emerges:

- The energy equation is

$$
\dot{m}_{l}^{\prime \prime} i_{l}+\dot{m}_{v}^{\prime \prime} i_{v}=k_{\text {eff }} \frac{d T}{d z}+\dot{q}^{\prime \prime} .
$$

- The mass conservation equation is

$$
\dot{m}_{l}^{\prime \prime}+\dot{m}_{u}^{\prime \prime}=\dot{m}^{\prime \prime},
$$

where $q^{\prime \prime}$ and $\dot{m}^{\prime \prime}$ are the unknown heat and mass fluxes (integration constants) that will be determined from the boundary conditions.

- The liquid momentum equation is

$$
\dot{m}_{l}^{\prime \prime}=-\rho_{l} \frac{K}{\mu_{l}} K_{r l}\left(\frac{d p_{c}}{d z}-\rho_{l} g \frac{L-h}{L}\right) .
$$

- The gas momentum equation is

$$
\dot{m}_{v}^{\prime \prime}+\dot{m}_{n}^{\prime \prime}=-\rho_{g} K \frac{k_{r g} d p}{\mu_{g}} \frac{d p_{g}}{d z},
$$

where $p_{g}$ is the total pressure of the gas mixture.

In Eqs. 23 and 24 we have modified the perme-

ability used by Whitaker [14] in order to recover the Darcy law for single phase flows. This is also noted by Whitaker [15].

- The gas diffusion equation is

$$
\left(\dot{m}_{v}^{\prime \prime}+\dot{m}_{n}^{\prime \prime}\right) y_{n}=\rho_{g} D_{\text {eff }} \frac{d y_{n}}{d z}
$$

In order to solve the governing equations, the constitutive equations found in Ref. 16 are used. These are as follows:

- Clapeyron-Kelvin equation (includes the effect of the meniscus curvature on the thermodynamic equilibrium state),

$$
\begin{aligned}
p_{g}= & \frac{p_{\text {sat }}}{1-y_{n}} \exp \left[\left(\frac{1}{T_{\text {sat }}}-\frac{1}{T}\right) \frac{i_{f g}}{R}\right. \\
& \left.-\frac{p_{v}-p_{l}}{\rho_{l} R T}\right] .
\end{aligned}
$$

- The equation of state (ideal gas behavior),

$$
p_{g}=\rho_{g} R T \text {. }
$$

- The capillary pressure (as a function of liquid saturation) is given by

$$
\begin{aligned}
p_{g}-p_{l}= & p_{c}=\frac{\sigma}{(K / \epsilon)^{1 / 2}}[1.42(1-S) \\
& \left.-2.12(1-S)^{2}+1.26(1-S)^{3}\right] \\
= & f \frac{\sigma}{(K / \epsilon)^{1 / 2}}
\end{aligned}
$$


where

$S=\frac{s-s_{\mathrm{im}}}{1-s_{\mathrm{im}}}$,

and the immobile saturation $s_{\text {im }}$ was estimated as 0.05 .

- The effective thermal conductivity is given by

$$
k_{\mathrm{eff}}=k_{s l}+s^{1 / 2}\left(k_{s l}+k_{s g}\right) \text {. }
$$

- The effective mass diffusivity is taken as

$$
D_{\mathrm{eff}}=\frac{\epsilon(1-s) D_{n v}}{\tau}
$$

The relative permeability of the porous medium is determined from the following approximations:

$$
K_{r l}=S^{3}, \quad K_{r g}=(1-S)^{3} .
$$

The gradient of the capillary pressure can be found by taking the total derivative of Eq. 28, i.e.,

$$
\frac{d p_{c}}{d z}=\frac{1}{(K / \epsilon)^{1 / 2}}\left(\sigma \frac{d f}{d s} \frac{d s}{d z}+f \frac{d \sigma}{d T} \frac{d T}{d z}\right)
$$

It should be noted that the surface tension gradient also influences the liquid flow by influencing the shear stress at the liquid-gas interface. This influence is not yet satisfactorily treated [17] and is not included here.

The boundary conditions are

$s+s_{o}, \quad T=T_{o}, \quad$ and $\quad y_{n}=y_{n, o}, \quad$ at

$z=0$,

$p_{g}=p_{\text {sat }}, \quad T=T_{\text {sat }}, \quad$ and $\quad \dot{m}^{\prime \prime}=\dot{m}_{b}^{\prime \prime}, \quad$ at

$z=L$,

where $p_{\text {sat }}$ is the pressure in the wind tunnel (assumed to be the atmospheric pressure). As shown in Ref. 18, four of the above boundary conditions are used to solve the initial value problems for $T, s, y_{n}$, and $p_{g}$. The last two are used to determine $\dot{q}^{\prime \prime}$ and $\dot{m}^{\prime \prime}$. The value of $s_{o}$ was selected such that the calculated temperature distribution would match the measured temperature. This was done through iteration, and, in general, we found $s_{o}$ to be approximately 0.83 .
Further discussion on two-phase flow and heat transfer in porous media can be found in Ref. 19.

The governing equations can be reduced to four initial value, first-order ordinary differential equations. The equations are solved by a fourthorder Runge-Kutta method. The specification of the values for $s, T, y_{n}$, and $p_{g}$ at $z=0$ is required. The net mass flow rate through the wick (which is equal to the burning rate) is obtained from the experiments, and a linear approximation is made to these results (Fig. 5). The details and solution steps are given in Ref. 18. The saturation distribution [including the surface saturation, i.e., $\left.s_{1}(z=L)\right]$ is found for various $\dot{m}(h)$. This leads to the relations between the burning rate and the surface saturation.

\section{RESULTS AND DISCUSSION}

Since we use the case of the flooded surface (a planar, thin liquid film present on the surface) as the baseline, and also in order to compare our measured burning rate with some existing measurements and predictions, experiments were also performed for $s_{1}=1$. In the following, the experimental results for $s_{1}$ are given first. Then, the experimental results for $s_{1}<1$ follow, where the predicted surface liquid saturation and the effect of this surface saturation on the burning rate (obtained through the diffusion model discussed in the section on mass diffusion) are presented, and the general behavior of $\dot{m}\left(s_{1}, \Delta / \lambda, \Delta / \delta, \operatorname{Re}\right)$

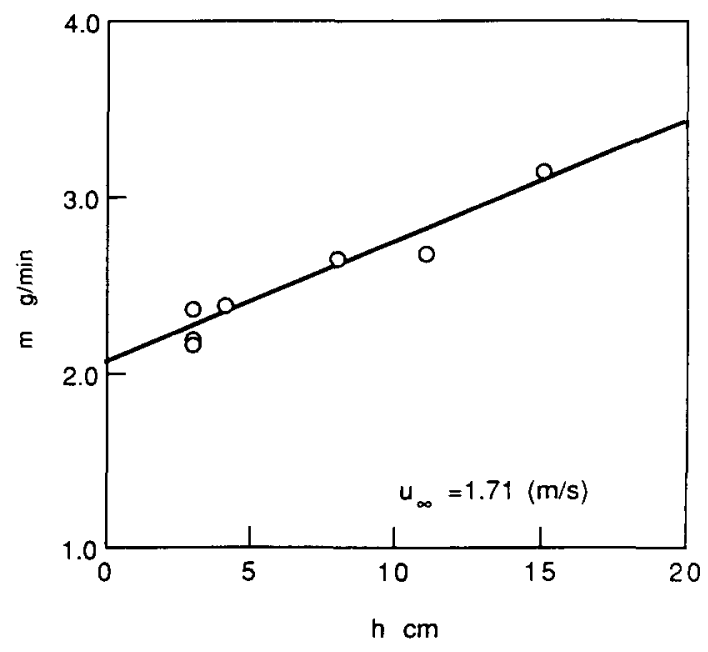

Fig. 5. Variation of the burning rate with respect to the hydrostatic pressure (height), for $u_{\infty}=1.71 \mathrm{~m} / \mathrm{s}$. 
TABLE I

Numerical Values for Parameters Appearing in the Analyses

\begin{tabular}{|c|c|c|}
\hline Parameters & Unit & Magnitude \\
\hline$B$ & & 3.26 \\
\hline$D_{n v}$ & $\mathrm{~m}^{2} / \mathrm{s}$ & $1.47 \times 10^{-5}$ \\
\hline$d \sigma / d T$ & $\mathrm{~N} / \mathrm{m}-\mathrm{K}$ & $-8.602 \times 10^{-5}$ \\
\hline$i_{f g}$ & $\mathrm{~J} / \mathrm{kg}$ & $8.8 \times 10^{5}$ \\
\hline$k_{g}$ & $\mathrm{~W} / \mathrm{m}-\mathrm{K}$ & 0.022 \\
\hline$k_{i}$ & $\mathrm{~W} / \mathrm{m}-\mathrm{K}$ & 0.155 \\
\hline$k_{s}$ & $\mathrm{~W} / \mathrm{m}-\mathrm{K}$ & 1.89 \\
\hline$k_{s g}$ & $\mathrm{~W} / \mathrm{m}-\mathrm{K}$ & 0.90 \\
\hline$k_{s \prime}$ & $\mathrm{W} / \mathrm{m}-\mathrm{K}$ & 1.012 \\
\hline$K^{3 \prime}$ & $\mathrm{m}^{2}$ & $8.5 \times 10^{-12}$ \\
\hline$l$ & $\mathrm{~m}$ & 0.043 \\
\hline$L$ & $\mathrm{~m}$ & 0.03 \\
\hline$P e$ & & 76.2 \\
\hline$R$ & $\mathrm{~J} / \mathrm{kg}-\mathrm{K}$ & 180.5 \\
\hline$T_{o}$ & $\mathbf{K}$ & 298.2 \\
\hline$T_{\text {sat }}$ & $\mathbf{K}$ & 351.5 \\
\hline$T_{\infty}$ & $\mathbf{K}$ & 297 \\
\hline$s_{\mathrm{im}}$ & & 0.05 \\
\hline$u_{\infty}$ & $\mathrm{m} / \mathrm{s}$ & 1.71 \\
\hline$\alpha_{I}$ & $\mathrm{~m}^{2} / \mathrm{s}$ & $5.36 \times 10^{-7}$ \\
\hline$\epsilon$ & & 0.5 \\
\hline$\mu_{g}$ & $\mathrm{~Pa}-\mathrm{s}$ & $1.1 \times 10^{-5}$ \\
\hline$\mu_{\prime}$ & $\mathrm{Pa}-\mathrm{s}$ & $5 \times 10^{-4}$ \\
\hline$\rho_{l}$ & $\mathrm{~kg} / \mathrm{m}^{3}$ & 770 \\
\hline $\bar{\sigma}$ & $\mathrm{N} / \mathrm{m}$ & 0.02 \\
\hline$\tau$ & & 2 \\
\hline
\end{tabular}

is examined. Table 1 gives the values of the properties and parameters used in this analysis.

\section{Burning Rate of Planar Liquid Surface \\ $\left(s_{1}=1\right)$}

Experiments were performed where the wick was placed in an aluminum casing with its upper surface placed about $3 \mathrm{~mm}$ below the top edge of this casing. Then the liquid fuel was allowed to totally fill this void by applying back pressure. The burning rate of this planar liquid surface was then measured. Figure 6 shows the experimental results, along with the experimental results of Ref. 20 and the prediction of Ref. 9. The latter can be expressed by the Glassman approximation [12] as

$\dot{m}^{\prime \prime}=\frac{\mu_{\infty}}{l} \frac{\ln (1+B)}{1.3 B^{0.15}} \operatorname{Re}_{l}^{0.5}$

Our measured $\dot{m}^{\prime \prime}$ is higher than the Emmons boundary-layer solution. This supports the argu-

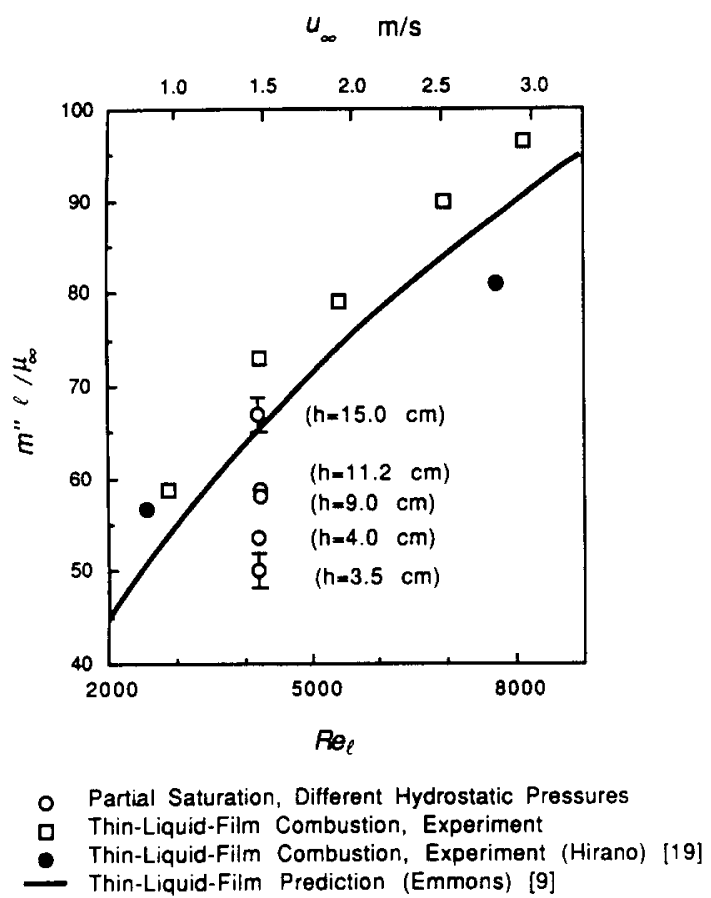

Fig. 6. Comparison of the experimental and predicted results for burning of a planar, thin-liquid-film surface. Also given are the experimental results for various partial liquid coverages and $u_{\infty}=1.71 \mathrm{~m} / \mathrm{s}$.

ment [21] that the burning rate obtained from the solution of the complete Navier-Stokes equations is higher than that based on the boundary-layer approximations. This is due to axial diffusion of species and heat at the leading edge. In the experiments, additional effects, not included in Emmons treatment, are also present. These are buoyancy, the slight surface indentation where the wick is imbedded, and the initiation of the momentum boundary layer upstream from the wick location. Note that the difference between the burning rate obtained from the boundary-layer solution and that obtained from the experiment is almost a constant.

\section{Burning Rate of Partially Liquid Covered Surface $\left(s_{1}<1\right)$}

Also shown in Fig. 6 are the results for $s_{1}<1$ and an ambient free-stream velocity of $1.71 \mathrm{~m} / \mathrm{s}$. At this velocity, the flame is stable and the buoyancy effect is small. The results show that the burning rate decreases as the hydrostatic pressure decreases (see also Fig. 5). 
The burning rate is controlled by capillaritygravity and by the hydrostatic pressure. A decrease in the hydrostatic pressure results in a decrease in the surface liquid saturation, which leads to an increase in the capillary pressure. If the increase in the capillary pressure compensates for the decrease in hydrostatic pressure, then the flow rate remains the same. As shown in Figs. 5 and 6 , this is not found here. Since the capillary pressure is strongly influenced by the microstructure of porous media, one can expect that the mean particle size of the wick (as a characteristic length) plays an important role among all the influencing factors. On the other hand, vapor diffusion through the indentations and the boundary-layer region is also affected by the mean wick particle size. The combined effects of the internal two-phase flow and the vapor diffusion near the surface determine the burning rate. These are discussed below.

\section{Surface Liquid Saturation}

The formulation for internal, two-phase, momentum, heat and mass transfer given in the previous section on surface liquid saturation yields the distribution of the liquid saturation $s$, gaseous pressure $p$, temperature $T$, and mass fraction of the noncondensible gas $y_{n}$ throughout the wick, including the surface. Figure 7 shows these distributions for $u_{\infty}=1.71 \mathrm{~m} / \mathrm{s}$. In the case where the hydrostatic pressure dominates the liquid flow (Fig. 7a for $h=9 \mathrm{~cm}$ ), the surface liquid saturation $s_{1}$ is just slightly less than saturation at the lower surface $s_{o}$ (note that $s_{1} \leq s_{o}$ ). When capillary pressure is important, i.e., when $(L-h) / L$ $\rightarrow 0$ (as shown in Fig. 7b), the negative, local saturation gradient causes the liquid motion towards the wick upper surface. This saturation gradient has a maximum absolute value at $z=L$, because the surface liquid saturation is smallest there. It should be noted that Eq. 32 includes the effect of the surface tension gradient as it influences the capillary pressure. When ethanol $d \sigma / d T<0$ and the temperature gradient is positive (Fig. 7), then the surface tension variation opposes the upward flow. For the hydrostaticdominated flows (Fig. 7a), this effect is small because of the weak influence of capillarity. When the capillary pressure is important (compared with the hydrostatic pressure) (Fig. 7b), the effect of

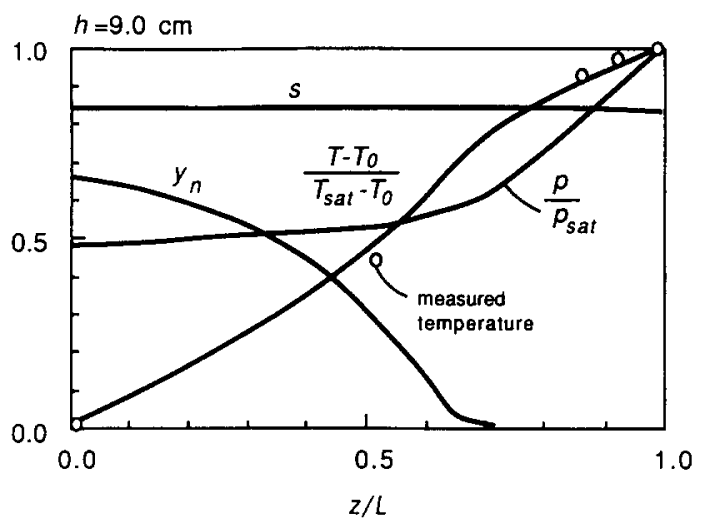

(a)

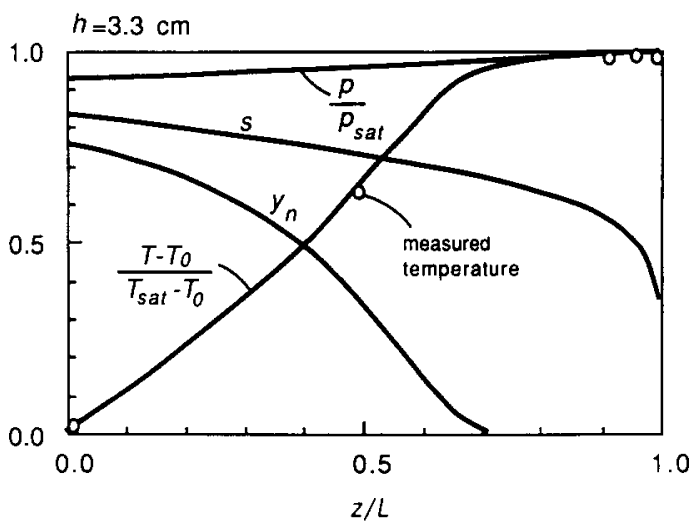

(b)

Fig. 7. Distributions of the temperature, saturation, mass fraction of the noncondensible gas, and gas pressure for $u_{\infty}=1.71 \mathrm{~m} / \mathrm{s}$. (a) Hydrostatically controlled flow $\left(s_{1} \approx s_{o}\right)$, (b) capillary and hydrostatically controlled flow $\left(s_{1}<s_{o}\right)$.

the surface tension gradient is also negligible where the temperature gradient is small.

Since the wick surface is permeable, a portion of the vapor produced by surface evaporation can flow back into the wick. This is through diffusion caused by the vapor concentration gradient induced by the temperature gradient in the wick. This also occurs through convection if a gaseous, total pressure gradient exists. As shown in Fig. 7, this inward vapor flow is most noticeable for high burning rates because of the large pressure and temperature gradients near the upper surface (Fig. 7a), but is not noticeable for the low burning rates (Fig. 7b). It can also be seen that the noncondensible gas is trapped near the cold surface of the wick $(z=0)$ as a consequence of this inward vapor flow. 


\section{Mass Diffusion Adjacent to Surface for $s_{1}<1$}

In the section on mass diffusion, we showed that vapor diffusion adjacent to the wick surface is greatly influenced by the ratio of the mean particle size to the concentration boundary-layer thickness. The effect of $\Delta / \lambda$ is not significant because for the problem considered, $\lambda$ is much smaller than $\Delta$. The predictions based on the development made above give the variation of the normalized mass transfer rate (Eq. 16) with respect to the surface saturation defined in Eq. 1 and $\bar{\delta} / d$. The results are shown in Figs. 8 and 9 for the conditions corresponding to the experiments.

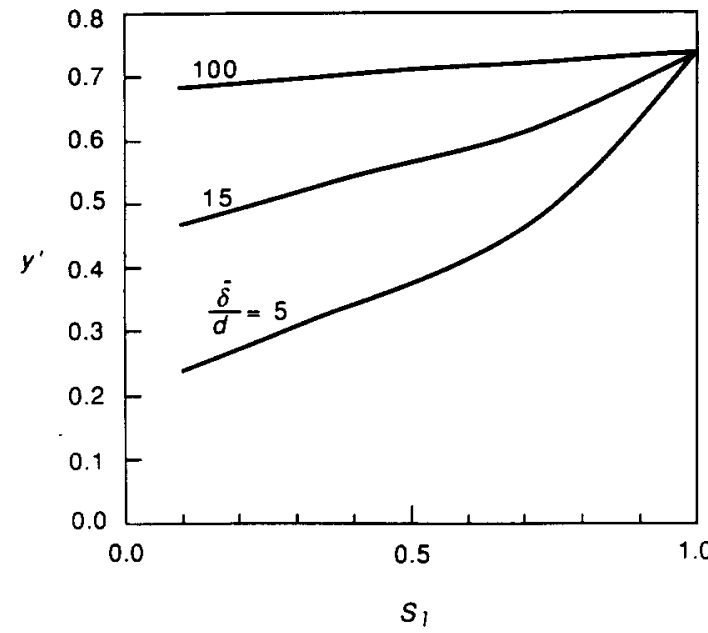

Fig. 8. Variation of the mass fraction of ethanol $y^{\prime}$ (at $Z=0$ ), with respect to the surface saturation, for several $\bar{\delta} / d$.

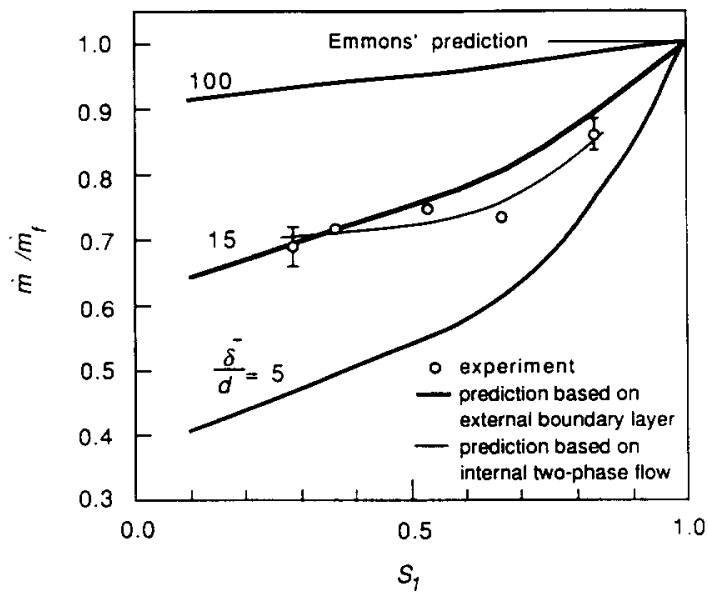

Fig. 9. Typical variation of the burning rate with respect to the surface saturation, for various $\bar{\delta} / d$.
As expected, the magnitude of $\bar{\delta} / d$ greatly influences the variation of the burning rate with respect to the liquid saturation. As shown in Fig. 8 , for $\bar{\delta} / d \geq 100$, the normalized burning rate has a magnitude of near unity, i.e., nearly independent of $s_{1}$. As $\bar{\delta} / d \rightarrow \infty$, the constant burning rate is reached, i.e., complete independence of $\dot{m} / \dot{m}_{f_{-}}$from $s_{1}$.

When $\bar{\delta} / d$ is finite (say less than 30 in the examples shown in Figs. 8 and 9), the dependence of the burning rate on the surface saturation becomes more pronounced. The normalized mass transfer rate, $\dot{m} / \dot{m}_{f}$, decreases rapidly with $s_{1}$ for $\bar{\delta} / d$ less than 10 . This indicates that the additional diffusion in a space ( $\Delta$ domain) caused by the surface protuberance/indentation is important for small values of $\bar{\delta} / d$ and can result in a significant reduction of the vapor mass fraction at the surface, $y^{\prime}(Z=0)$. This observation is supported by a qualitative comparison of the prediction (based on one-dimensional internal and external models) with the experimental results, shown in Fig. 9. The experimental results are close to the predictions for $\bar{\delta} / d=15$. If we estimate the concentration boundary-layer thickness for the experimental condition $\left(\mathrm{Re}_{l}=4200\right.$, $l=0.043 \mathrm{~m}$ ), using the Blasius approximation and assuming $\mathrm{Sc}=1$, we have

$\bar{\delta}=\frac{l 5.2}{2} \operatorname{Re}_{l}^{-1 / 2}$,

which gives $\bar{\delta}=1.7 \mathrm{~mm}$. The Carman-Kozeny relation (based on nonconsolidated spherical particles) gives the average particle size of the ceramic wick as $55 \mu \mathrm{m}$. Therefore, from the experiments, we have $\bar{\delta} / d \simeq 27$. The differences in $\bar{\delta} / d$ between the predictions and the experiments is mainly because the wick is not made of nonconsolidated spherical particles and may have a larger equivalent mean particle size than that predicted from the Carman-Kozeny relation. Despite this difference, the above comparison points out, from a mass diffusion point of view, the importance of the effect of the surface saturation on the burning rate for small $\bar{\delta} / d$. Since $\bar{\delta}$ is inversely proportional to the Reynolds number (to the power -0.5 for laminar flows), the liquid saturation dependence of the burning rate would be more pronounced at high Reynolds numbers.

We can also view the influence of vapor diffu- 
sion in the $\Delta$ domain on the burning rate in terms of a total resistance to diffusion, $R$, where $R$ is defined such that $\dot{m} / \dot{m}_{f}=\left(R / R_{f}\right)^{-1}$. From Eq. 14 , we arrive at the following approximation (assuming $\ln (1+B) \approx B$ ):

$$
\frac{R}{R_{f}} \sim \frac{d}{\bar{\delta}}, \quad \text { for fixed } B^{*} \text { and } s_{1} \text {, }
$$

and

$$
\frac{R}{R_{f}} \sim B^{* 0.85}, \quad \text { for fixed } \frac{\bar{\delta}}{d} \text { and } s_{1} \text {. }
$$

Then, as the ratio $\bar{\delta} / d$ decreases or as $B^{*}$ increases, the total resistance to the diffusion increases, resulting in a decrease in the mass transfer rate. The first relation above is consistent with the results shown in Fig. 9. The second relation suggests that using fuels with high mass transfer numbers may result in larger reduction in the burning rate (for a given wick and surface saturation).

In the vapor diffusion model given in the mass diffusion section, the heat penetration into the wick, which is a form of heat loss, was neglected. This heat loss, which is due to the subcooled state of the liquid supplied at the lower wick surface, can be accounted for by modifying the mass transfer number as [9]

$$
B^{\prime}=\frac{c_{p}\left(T_{\infty}-T^{\prime}\right)+\beta y_{O, \infty} Q}{i_{f g}+Q_{c}},
$$

where $Q_{c}$ is the heat penetration through the wick, which is a function of the surface liquid saturation. Our calculation indicates that $Q_{c}$ increases slightly as $s_{1}$ decreases. Inclusion of $Q_{c}$ reduces the value of $B^{\prime}$, and, therefore, reduces the predicted burning rate. For the typical case shown in Fig. 5, $Q_{c}$ is less than $10 \%$ of the heat for the evaporation of ethanol at the upper wick surface. This gives a reduction of $10 \%$ in $B^{\prime}$. However, since the liquid fuel used in the experiment for the fully liquid covered surface is also subcooled, we have a reduction in $B^{*}$ that is the same order of that in $B^{\prime}$. From Eq. 9, it can be seen that these reductions may cancel each other (if they are the same) in the expression of the normalized mass transfer rate $\dot{m} / \dot{m}_{f}$. Our calculation indicates that the difference in the predicted $\dot{m} / \dot{m}_{f}$ caused by including $Q_{c}$ is less than $2 \%$. This shows that in our experiments, the effect of the surface roughness is more important than that of $Q_{c}$ because the effective thermal conductivity of the ceramic wick used is rather small (Table 1). For wicks with larger $k_{\text {eff }}$ (e.g., metallic wicks), this subcooling effect becomes significant. The exposed solid matrix transfers more heat from the ambient gas to the liquid. Therefore, the burning rate can remain the same or even increase as the surface saturation decreases. This effect of thermal conductivity of the solid matrix on the burning rate has been observed experimentally [18].

\section{CONCLUSIONS}

For liquid fuels supplied through wicks, the vapor pressure at the surface being burned is high enough so that the ratio of the characteristic length of the exposed matrix (i.e., the surface roughness) is much larger than the mean free path of the vapor molecule. However, the ratio of this characteristic length to the boundary-layer thickness (momentum, thermal, and concentration) may not be small, especially when the flow is at least partly due to capillarity. Then, our results show that the burning rate for partially exposed wick matrices is less than that for fully liquid covered surfaces. The permeability of $8.5 \times$ $10^{-12}\left(\mathrm{~m}^{2}\right)$ and porosity of 0.5 are typical of wicks that offer large enough capillary pressure (especially, suitable for surface orientations other than horizontal) and yet their resistance to liquid flow is not so large as to require an external driving pressure. Therefore, we expect some deviations from the ideal behavior of planar, liquid-film-covered surfaces when these wicks are used as simulants. We also mentioned that the ceramic wick used here has an effective thermal conductivity that is much closer to that of the liquid than some of the metallic wicks that have been used as simulants. In the case of metallic wicks, the exposed matrix of high thermal conductivity tends to increase the burning rate. Therefore, partial-liquid-covered metallic wicks can, under certain conditions, have burning rates as high as or even higher than that for the planarthin-liquid film covered surfaces.

\section{REFERENCES}

1. Ahmed, T., and Faeth, G. M., J. Heat Transf. 100:112-119 (1978). 
2. Groff, E. G., and Faeth, G. M., Combust. Flame 32:139-150 (1978).

3. Malary, S. F., Awad, J. K., and Thapar, R., Combust. Sci. Technol. 63:1-11 (1989).

4. Tao, Y.-X., and Kaviany, M., J. Heat Transf. (to appear).

5. Rogers, J. A., and Kaviany, M., J. Heat Transf. 112:668-674 (1990).

6. Schlünder, E. U., Chem. Eng. Sci. 43:2685-2688 (1988).

7. Kaviany, M., and Tao, Y.-X., J. Heat Transf. 110:431-436 (1988).

8. Kim, J. S., de Ris, J., and Kroesser, F. W., Thirteenth Symposium (International) on Combustion, The Combustion Institute, Pittsburgh, 1970, pp. 949-961.

9. Emmons, H. W., Z. Angew. Math. Mech. 36:60-71 (1956).

10. Kaviany, M., and Mittal, M., Int. J. Heat Mass Transf. 30:1407-1418 (1987).

11. Tien, C. L., and Lienhard, J. H., Statistical Thermodynamics, Holt, Rinehart, and Winston, New York. 1971 , p. 317.

12. Glassman, I., Combustion, Academic, New York, 1987, pp. 281-287.
13. Leverett, V. G., Trans. AIME 142:152-169 (1941).

14. Whitaker, S., in Advances in Heat Transfer (J. P. Hartnett, and T. F. Irvine, Jr., Ed.), Academic, New York, 1977, Vol. 13, pp. 119-203.

15. Whitaker, S., Transp. Porous Media 1:105-125 (1986).

16. Udell, K. S., Int. J. Heat Mass Transf. 28:485-495 (1985).

17. Bear, J., and Bensabat, J., Transp. Porous Media 4:423 (1989).

18. Tao, Y.-X., Ph. D. dissertation, University of Michigan, 1989.

19. Kaviany, M., Principles of Heat Transfer in Porous Media, a monograph to be published by Springer-Verlag in the Mechanical Engineering Series, 1991.

20. Hirano, T., and Kinoshita, M., Fifteenth Symposium (International) on Combustion, The Combustion Institute, Pittsburgh, 1974, pp. 379-387.

21. Chen, C.-H., and T'ien, J. S., Combust. Sci. Technol. 50:283-306 (1986).

Received 24 May 1990; revised 18 February 1991 